https://doi.org/10.15407/ujpe64.8.702

S.A. LUKASHEVICH, N.V. MAKSIMENKO

Francisk Skorina Gomel State University

(98, Sovetskaya Str., Gomel 246019, Belarus; e-mail: lukashevich@gsu.by)

\title{
THE CONSTRUCTION OF RELATIVISTICALLY INVARIANT EQUATIONS OF MOTION AND THE MOMENTUM ENERGY TENSOR FOR SPIN-1/2 PARTICLES WITH POLARIZABILITIES IN AN ELECTROMAGNETIC FIELD
}

Within the covariant Lagrangian formalism, the equations of motion for spin-1/2 particles with polarizabilities in an electromagnetic field have been obtained. We have analyzed the phenomenological tensor constant quantities as well.

Ke ywords: covariant Lagrangian, equations of motion, energy-momentum tensor.

\section{Introduction}

The interaction of an electromagnetic field with structural particles in the electrodynamics of hadrons is based on the main principles of relativistic quantum field theory. In the model conceptions, where basically the diagram technique is used, a number of features for the interaction of photons with hadrons have been determined $[1,2]$. However, the diagram technique is mainly employed for the description of electromagnetic processes in the simplest quark systems. In the case of interaction for the electromagnetic field with complex quark-gluon systems in the low-energy region, the perturbative methods of QCD are nonapplicable. That is why, the low-energy theorems and sum rules were widely used lately [3-6]. In the present time, the low-energy electromagnetic characteristics which are connected with a hadron structure, such as the formfactor and polarizabilities, can be obtained from nonrelativistic theory [5]. Passing from the nonrelativistic electrodynamics to the relativistic field theory, one can use the correspondence principle. But it is necessary to investigate, step-by-step, a transition from the covariant Lagrangian formalism to the Hamiltonian one [7-9].

The determination of the interaction vertex of $\gamma$ photons with protons taking the polarizabilities into account [10] has recently been used to fit experimental data on the Compton scattering on a proton in

(c) S.A. LUKASHEVICH, N.V. MAKSIMENKO, 2019 the energy neighborhood of a birth of the $\Delta(1232)$ resonance [11].

This work is a continuation of the researches which have been presented in our previous articles [6-8]. Using the covariant Lagrangian of interaction of the electromagnetic field with a structural polarizable particle, the equations of motion and the canonical and metric energy-momentum tensors have been obtained.

\section{Total Lagrangian}

The total Lagrangian of the interaction of spin- $1 / 2$ particles with the electromagnetic field consists of the Lagrangian for the free electromagnetic field $L_{e-m}$, the spinor or Dirac field $L_{D}$, the Lagrangian of the interaction of the free electromagnetic field with the Dirac field $L_{\text {int }-D}$, and the Lagrangian which considers electric and magnetic polarizabilities of particles $L_{\alpha_{0} \beta_{0}-D}$ :

$L_{\text {total }-D}=L_{e-m}+L_{D}+L_{\text {int }-D}+L_{\alpha_{0} \beta_{0}-D}$,

thus,

$L_{\text {total }-D}=-\frac{1}{4} F_{\alpha \beta} F^{\alpha \beta}+\bar{\psi}\left(\frac{1}{2} i \gamma_{\alpha} \stackrel{\leftrightarrow}{\partial^{\alpha}}-m\right) \psi-$

$-e\left(\bar{\psi} \gamma_{\alpha} \psi\right) A^{\alpha}+K_{\sigma \nu} \Theta^{\sigma \nu}$

where

$K_{\sigma \nu}=\frac{2 \pi}{m}\left(\alpha_{0} F_{\sigma \mu} F_{\nu}^{\mu}+\beta_{0} \tilde{F}_{\sigma \mu} \tilde{F}_{\nu}^{\mu}\right)$,

$\overleftrightarrow{\partial_{\nu}}=\overleftarrow{\partial_{\nu}}-\overrightarrow{\partial_{\nu}}$

ISSN 2071-0194. Ukr. J. Phys. 2019. Vol. 64, No. 8 
$\Theta^{\sigma \nu}=\frac{i}{2}\left(\bar{\psi} \gamma^{\sigma} \stackrel{\leftrightarrow}{\partial^{\nu}} \psi\right)$

$\psi$ is the wave function of spin- $1 / 2$ particles.

In this expression $\tilde{F}_{\mu \nu}=\frac{1}{2} \varepsilon_{\mu \nu \rho \sigma} F^{\rho \sigma}$, where $F_{\mu \nu}$ and $\tilde{F}_{\mu \nu}$ are the tensors of the electromagnetic field, $\alpha_{0}$ and $\beta_{0}$ are electric and magnetic polarizabilities, and $\varepsilon_{\mu \nu \rho \sigma}$-Levi-Civita antisymmetric tensor $\left(\varepsilon^{0123}=1\right)$.

The part of the Lagrangian with polarizabilities can be rewritten as

$L^{(\alpha \beta)}=-\frac{1}{4} F_{\mu \nu} G^{\mu \nu}=K_{\sigma \nu} \Theta^{\sigma \nu}$,

where $G^{\mu \nu}$ is the antisymmetric tensor $G^{\mu \nu}=-G^{\nu \mu}$ and is equal to

$G^{\mu \nu}=-\frac{\partial L^{(\alpha \beta)}}{\partial\left(\partial_{\mu} A_{\nu}\right)}=\frac{4 \pi}{m}\left(\left(\alpha_{0}+\beta_{0}\right)\left(F_{\sigma}^{\mu} \widetilde{\Theta}^{\rho \nu}-\right.\right.$

$\left.\left.-F_{\rho}^{\nu} \widetilde{\Theta}^{\rho \mu}\right)-\beta_{0} \Theta_{\rho}^{\rho} F^{\mu \nu}\right)$,

where

$\widetilde{\Theta}^{\rho \nu}=1 / 2\left(\Theta^{\rho \nu}+\Theta^{\nu \rho}\right)$.

\section{Equations of Motion}

For the interaction of the spinor and electromagnetic fields, the following system of equations is used:

$-\frac{\partial L}{\partial A_{\mu}}+\partial_{\gamma} \frac{\partial L}{\partial\left(\partial_{\gamma} A_{\mu}\right)}=0$

$-\frac{\partial L}{\partial \bar{\psi}}+\partial_{\gamma} \frac{\partial L}{\partial\left(\partial_{\gamma} \bar{\psi}\right)}=0$

$-\frac{\partial L}{\partial \psi}+\partial_{\gamma} \frac{\partial L}{\partial\left(\partial_{\gamma} \psi\right)}=0$

where $A_{\mu}$ is the vector-potential of the electromagnetic field.

From Lagrangian (1) and expressions (4-6), we get the equations of motion for a charged spin- $1 / 2$ particle with $\alpha_{0}$-electric and $\beta_{0}$-magnetic polarizabilities:

$$
\begin{aligned}
& \partial_{\mu} F^{\mu \nu}=e \bar{\psi} \gamma^{\nu} \psi-\partial_{\mu} G^{\mu \nu}, \\
& \left(i \gamma^{\nu} \vec{\partial}_{\nu}-m\right) \psi=e A_{\nu} \gamma^{\nu} \psi-\frac{i}{2}\left(\partial^{\nu} K_{\sigma \nu} \gamma^{\sigma}\right) \psi- \\
& -i K_{\sigma \nu} \gamma^{\sigma} \partial^{\nu} \psi, \\
& \bar{\psi}\left(i \overleftarrow{\partial_{\nu}} \gamma^{\nu}+m\right)=-e \bar{\psi} A_{\nu} \gamma^{\nu}- \\
& -\frac{i}{2} \bar{\psi}\left(\partial^{\nu} K_{\sigma \nu} \gamma^{\sigma}\right)-i\left(\partial^{\nu} \bar{\psi}\right) \gamma^{\sigma} K_{\sigma \nu} .
\end{aligned}
$$

ISSN 2071-0194. Ukr. J. Phys. 2019. Vol. 64, No. 8
In expression (7),e $\bar{\psi} \gamma^{\nu} \psi$ is the current associated with a charge, $-\partial_{\mu} G^{\mu \nu}$ is the current associated with the polarizabilities of the particle.

Following work [12], we perform a relativistic generalization of the phenomenological energy-momentum tensor of the interaction of the electromagnetic field with a polarizable particle as

$T^{\mu \nu}=T_{0}^{\mu \nu}+T_{(\alpha \beta) \text { int }}^{\mu \nu}$

Lagrangian (1) takes the form

$L_{\text {total }-D}=L_{0}+L_{\text {int }}$,

where

$L_{0}=-\frac{1}{4} F_{\alpha \beta} F^{\alpha \beta}+\bar{\psi}\left(\frac{1}{2} i \gamma_{\alpha} \stackrel{\leftrightarrow}{\partial^{\alpha}}-m\right) \psi$

is the usual Lagrangian, and

$L_{\mathrm{int}}=-e\left(\bar{\psi} \gamma_{\alpha} \psi\right) A^{\alpha}+K_{\sigma \nu} \Theta^{\sigma \nu}$

is the interaction Lagrangian of the electromagnetic field and a particle with polarizabilities.

With the help of Lagrangian (11), the canonical energy-momentum tensor looks like

$T_{\text {can }}^{\mu \nu}=\frac{\partial L_{0}}{\partial\left(\partial_{\mu} A_{\rho}\right)}\left(\partial^{\nu} A_{\rho}\right)+\partial^{\nu} \bar{\psi} \frac{\partial L_{0}}{\partial\left(\partial_{\mu} \bar{\psi}\right)}+\frac{\partial L_{0}}{\partial\left(\partial_{\mu} \psi\right)} \partial^{\nu} \psi-$ $-g^{\mu \nu}\left(-\frac{1}{4} F_{\alpha \beta} F^{\alpha \beta}-\frac{1}{4} F_{\alpha \beta} G^{\alpha \beta}\right)$.

As a result, we get

$T_{\mathrm{can}}^{\mu \nu}=T_{\mathrm{can}(0)}^{\mu \nu}+\frac{g^{\mu \nu}}{4} G_{\rho \sigma} F^{\rho \sigma}$,

where $\frac{g^{\mu \nu}}{4} G_{\rho \sigma} F^{\rho \sigma}$ is the energy-momentum tensor of the interaction of the electromagnetic field with regard for the polarizabilities of the particle, and

$T_{\operatorname{can}(0)}^{\mu \nu}=-F^{\mu \rho} \partial^{\nu} A_{\rho}+\frac{g^{\mu \nu}}{4} F_{\rho \sigma} F^{\rho \sigma}+\Theta^{\mu \nu}$.

Using the unambigious definition of a energymomentum tensor for $T_{\text {can }}^{\mu \nu}$, we construct the metric energy-momentum tensor:

$T_{\mathrm{metr}}^{\mu \nu}=T_{\mathrm{can}(0)}^{\mu \nu}+\partial_{\rho}\left(F^{\mu \rho} A^{\nu}\right)+\frac{g^{\mu \nu}}{4} G_{\rho \sigma} F^{\rho \sigma}$.

Thus, $T_{\text {metr }}^{\mu \nu}$ reads

$T_{\text {metr }}^{\mu \nu}=F^{\mu \rho} F_{\rho}^{\nu}+\frac{g^{\mu \nu}}{4} F_{\rho \sigma} F^{\rho \sigma}+\Theta^{\mu \nu}-$ 
$-j^{\mu} A^{\nu}+\frac{g^{\mu \nu}}{4} G_{\rho \sigma} F^{\rho \sigma}$,

where $j^{\mu}$ is the current density of the charged particle.

In the rest frame of the particle, we obtain the energy density of interaction for the particle with polarizabilities and the electromagnetic field:

$\mathcal{E}=-\frac{2 \pi}{m} \Theta^{00}\left(\alpha_{0} \mathbf{E}^{2}+\beta_{0} \mathrm{H}^{2}\right)$,

where $\Theta^{00}$ is the energy density of the spin- $1 / 2$ particle.

\section{Conclusion}

Taking the covariant Lagrangian of interaction of the electromagnetic field with a polarizable spin- $1 / 2$ particle as a basis in the Lagrangian covariant formalism, the equations of motion have been found. The correlations between the covariant Lagrangian and the canonical and metric energy-momentum tensors have been obtained. In the rest frame of the particle, the energy density of interaction for the particle with polarizabilities and the electromagnetic field has been determined.

1. S.J. Brodsky, J.R. Primack. The electromagnetic interaction of composite systems. Annals of Physics 52, 315 (1969).

2. S. Scherer, A. Yu. Korchin, J.H. Koch. Virtual Compton scattering off the nucleon at low energies. Phys. Rev. C 54, 904 (1996).

3. M.I. Levchuk, L.G. Moroz. Gyration nucleon as one of the characteristics of its electromagnetic structure. Vesti AN BSSR Ser.: fiz.-mat. nauk 1, 49 (1985).

4. A.J. L'vov, V.A. Petrun'kin. Dispersion theory of proton Compton scattering in the first and second resonance regions. Phys. Rev. C 55, 359 (1997).
5. M.-Th. Hutt, A.J. L'vov, A.J. Milstein, M. Schumacher. Compton scattering by nuclei. Phys. Reports 323, No. 6, 458 (2000).

6. N.V. Maksimenko, L.G. Moroz. Phenomenological description polarizabilities of elementary particles in a fieldtheory. In: Proc. 11 Intern. School on High Energy Physics and Relativistic Nucl. Phys. Dubna JINR D2-11707, 533 (1979).

7. S.A. Belousova, N.V. Maksimenko. The description for spin polarizabilities based on the covariant Lagrangian. In Proc. of "OFTHEP'2000". Tver, Russia, 305 (2000), hep$\mathrm{ph} / 0009334$.

8. N.V. Maksimenko, O.M. Deruzhkova, S.A. Lukashevich. The electromagnetic characteristics of hadrons in the covariant Lagrangian approach. In Proc. of Intern. SchoolSeminar "Actual Problems of Particles Physics", Gomel, 2001. JINR 2, 145 (2002).

9. D. Babusci, J. Jiordano, A.J. L'vov, J. Matone, A.N. Nathan. Low-energy Compton scattering of polarized photons on polarized nucleons. Phys. Rev. C 58, 1013 (1998).

10. A. Ilyichev, S. Lukashevich, N. Maksimenko. Static polarizability vertex and its application. arXiv: hep$\mathrm{ph} / 0611327 \mathrm{v} 1$.

11. Y. Zhang, K. Savvidy. Proton Compton scattering in a unified proton $\Delta^{+}$model. Phys. Rev. C 88, No. 6, 064614 (2013). arXiv:1305.3847 [hep-ph] NITS-PHY-2013002.

12. C. Itzykson, J.B. Zuber. Quantum Field Theory (McGrawHill, 1980).

Received 08.07.19

С.А. Лукашевич, Н.В. Максименко

ПОБУДОВА

РЕЛЯТИВІСТСЬКО-ІНВАРІАНТНИХ

РІВНЯНЬ РУХУ ТА ТЕНЗОР

ЕНЕРГІЇ-ІМПУЛЬСУ ДЛЯ ЧАСТИНОК

ЗІ СПІНОМ 1/2 3 ПОЛЯРИЗОВНІСТЮ

В ЕЛЕКТРОМАГНІТНОМУ ПОЛІ

Р е з ю м е

В рамках коваріантного лагранжового формалізму отримано рівняння руху для частинок зі спіном $1 / 2$ з поляризовністю в електромагнітному полі. Нами також проаналізовано феноменологічні тензорні константи. 